\title{
Roles of flavonoids and the transcriptional regulator Ttsl in the activation of the type III secretion system of Bradyrhizobium elkanii SEMIA587
}

\begin{abstract}
Correspondence
Luciane M. P. Passaglia

Ipassaglia@terra.com.br
\end{abstract}

Received 22 April 2010

Revised 9 November 2010

Accepted 19 November 2010

\author{
Samanta Bolzan de Campos, ${ }^{1}$ William J. Deakin, ${ }^{2}$ William J. Broughton ${ }^{2}$ \\ and Luciane M. P. Passaglia ${ }^{1}$
}
${ }^{1}$ Universidade Federal do Rio Grande do Sul, Instituto de Biociências, Departamento de Genética, Av. Bento Gonçalves, 9500, Caixa Postal 15.053, CEP 91501-970, Porto Alegre, RS, Brazil

${ }^{2}$ Laboratoire de Biologie Moléculaire des Plantes Supérieures (LBMPS), Département de Biologie Végétale, Sciences III, 30 Quai Ernest-Ansermet, University of Geneva, CH-1211 Geneva 4, Switzerland

\begin{abstract}
Bradyrhizobium elkanii SEMIA587 is a symbiotic nitrogen-fixing bacterium of the group commonly called rhizobia, which induce nodule formation in legumes, and is widely used in Brazilian commercial inoculants of soybean. In response to flavonoid compounds released by plant roots, besides Nod factors, other molecular signals are secreted by rhizobia, such as proteins secreted by type III secretion systems (T3SSs). Rhizobial T3SSs are activated by the transcription regulator Ttsl, which binds to sequences present in the promoter regions of T3SS genes via a conserved sequence called the $t$ ts box. To study the role of the T3SS of $B$. elkanii SEMIA587, tts/ was mutated. Protein secretion and flavonoid induction analysis, as well as nodulation tests, were performed with the wild-type and mutant strains. The results obtained showed that B. elkanii SEMIA587 secretes at least two proteins (NopA and NopL, known rhizobial T3SS substrates) after genistein induction, whilst supernatants of the tts/ mutant did not contain these Nops. Unusually for rhizobia, the promoter region of the B. elkanii SEMIA587 tts/ gene contains a tts box, which is responsive to flavonoid induction and to which Ttsl can bind. Nodulation tests performed with three different leguminous plants showed that the $B$. elkanii SEMIA587 tts/ mutant displays host-dependent characteristics; in particular, nodulation of two soybean cultivars, Peking and EMBRAPA 48, was more efficient when Ttsl of B. elkanii was functional.
\end{abstract}

\section{INTRODUCTION}

Type III secretion systems (T3SSs) are specialized machines for protein secretion used by many Gram-negative bacteria. The secretion machinery is formed by about 20 proteins, many of which are homologous to those of the apparatus for flagella assembly. When not used for flagella assembly, T3SSs function to deliver proteins (called effectors) directly into the cytosol of eukaryotic cells (Pallen et al., 2003). T3SSs were first thought to be exclusive to pathogenic bacteria (Hueck, 1998), but after sequencing the symbiotic plasmid of Rhizobium sp. NGR234 (hereafter NGR234), sequences homologous to all the genes encoding a T3SS and to possible effectors were found (Freiberg et al., 1997). The NGR234 T3SS is functional and involved in the

Abbreviations: EMSA, electrophoretic mobility shift assay; GST, glutathione S-transferase; T3SS, type III secretion system.

The GenBank/EMBL/DDBJ accession number for the $t t s /$ coding region sequence of $B$. elkanii is HM122010. determination of the host range of nodulation by NGR234 (Viprey et al., 1998). Proteins secreted by rhizobial T3SSs are termed nodulation outer proteins (Nops) (Marie et al., 2001), and can have different roles, depending on the host plant. Some effector Nops can enhance nodulation in specific leguminous plants, whilst in others Nops may be recognized as virulence factors, decreasing nodule formation (Viprey et al., 1998; Krishnan et al., 2007; Bartsev et al., 2003, 2004; Skorpil et al., 2005).

In rhizobia, T3SSs are activated by NodD1 in a flavonoiddependent manner. NodD1 activates the expression of the transcriptional activator TtsI by binding to the nod box in the promoter region of its gene $(t t s I)$. A nod box element has been identified in the promoters of all ttsI genes studied (Viprey et al., 1998; Krause et al., 2002; Marie et al., 2003, 2004; López-Baena et al., 2008; Wassem et al., 2008). No nod box sequences are found in the promoter regions of other genes encoding T3SSs; instead, a different cis element has been identified and named the $t$ ts box by Krause et al. 
(2002). TtsI activates the transcription of other T3SSrelated genes by binding to the consensus $t t s$ box sequence nnntcGTCAGcttntcGaaAGctagnccnntannnnn (Krause et al., 2002; Marie et al., 2004; López-Baena et al., 2008; Wassem et al., 2008; Zehner et al., 2008). Copies of this consensus sequence have been found in the promoter regions of the T3SS genes of NGR234 (Marie et al., 2004; Wassem et al., 2008), Bradyrhizobium japonicum USDA110 (Krause et al., 2002; Zehner et al., 2008), Sinorhizobium fredii HH103 and S. fredii USDA257 (López-Baena et al., 2008; Krause et al., 2002).

TtsI has homology to the DNA-binding response regulators of two-component regulatory systems (Viprey et al., 1998; Marie et al., 2004; Wassem et al., 2008). Such regulators are usually activated by their cognate sensor kinase, which transfers a phosphoryl group to an aspartate residue in the regulator, leading to a conformational change that activates the latter protein. Once phosphorylated, the regulator protein binds to cis elements located in the promoter region of genes that are required to process the environmental signal detected by the sensor partner. TtsI, however, has a glutamate residue instead of the usual conserved aspartate. It has already been shown that this type of substitution leads to a constitutive activation of the regulator protein, without the need of its partner sensor kinase (Lan \& Igo, 1998). It has been proposed that TtsI acts as a transcriptional activator independently of phosphorylation by the sensor kinase (Marie et al., 2004; Wassem et al., 2008). Thus, transcription of T3SS genes would be dependent on the NodD1 activation of the $t t s I$ gene, in the presence of the specific flavonoid compound released by the host plant (Kobayashi et al., 2004; Marie et al., 2004; Wassem et al., 2008).

Nodulation of soybean can be induced by various rhizobial strains that possess a T3SS. Mutation of the T3SS of $B$. japonicum USDA110 leads to a delay in nodule formation by Glycine max cultivar Williams (Krause et al., 2002). Similarly, a functional T3SS in S. fredii $\mathrm{HH} 103$ enhances nodulation of various soybean cultivars (López-Baena et al., 2008). The T3SS of $S$. fredii USDA257 has varying effects on the nodulation of soybean cultivars. With cultivar Peking, an active T3SS improves nodulation, whereas the T3SS must be mutated to allow nodulation of cultivar McCall (Meinhardt et al., 1993).

In Brazil, Bradyrhizobium elkanii is successfully used in the formulation of commercial inoculants to increase soybean yields and, together with $B$. japonicum, fully supplies plant nitrogen demands. At present, only four B. japonicum and $B$. elkanii strains are routinely used in the formulation of Brazilian commercial inoculants, and they have resulted in an established rhizobial population in most soils cropped with soybean (Ferreira et al., 2000). Of the recommended strains, SEMIA587 and SEMIA5019 belong to the species $B$. elkanii, whilst SEMIA5080 and SEMIA5079 belong to the species B. japonicum (Rumjanek et al., 1993).

Genomic hybridization performed by Viprey et al. (1998) has shown that T3SS homologues are present in B. elkanii
USDA76. A $47.1 \mathrm{~kb}$ DNA sequence containing a T3SS cluster from B. elkanii USDA61 was recently obtained by Okazaki et al. (2009). The creation of T3SS mutants of this strain has shown that it has a host-dependent nodulation phenotype: a functional T3SS improves nodulation of cultivar Clark but blocks nodulation of cultivar Hill (Okazaki et al., 2009). Characterization of the DNA sequence in the T3SS locus of B. elkanii USDA61 has shown that it possesses ttsI (preceded by a nod box) as well as numerous $t t s$ boxes upstream of key genes encoding T3SS components. Despite containing all the elements required for flavonoid-based induction of protein secretion, this has not been observed and the presence of flavonoids in the growth medium has no effect on protein secretion (Okazaki et al., 2009).

In this work we investigated the role of the T3SS in the agronomically important Brazilian (soybean) inoculant B. elkanii SEMIA587 (hereafter SEMIA587). Identification of T3SS-related sequences permitted the creation of a ttsI mutant and thus an inactive T3SS. The effects on the nodulation of soybean cultivars (and other legumes) known to differentially respond to rhizobial T3SSs, as well as on a Brazilian soybean cultivar, were assessed. Characterization of the SEMIA587 TtsI showed it (and hence the T3SS) to be induced by flavonoids produced by soybean.

\section{METHODS}

Bacterial cultures and growth conditions. SEMIA587 and its derivatives were grown in YEM (yeast extract mannitol) (Vincent, 1970) and RMS (Rhizobium minimal medium containing succinate as the carbon source; Broughton et al., 1986) media at $28^{\circ} \mathrm{C}$. Escherichia coli strains DH5 $\alpha$, BL21 (Rosetta) and XL1-Blue were grown in LuriaBertani (LB) broth at $37{ }^{\circ} \mathrm{C}$ (Sambrook \& Russell, 2001). Antibiotics were added to the media at the following final concentrations: tetracycline, $50 \mu \mathrm{g} \mathrm{ml}{ }^{-1}$; kanamycin, $50 \mu \mathrm{g} \mathrm{ml}^{-1}$; spectinomycin, $100 \mu \mathrm{g} \mathrm{ml}^{-1}$. Where indicated, cultures were induced with genistein or daidzein at a final concentration of $10^{-6} \mathrm{M}$. Control cultures were treated with the corresponding amount of methanol, which was used as the solvent for these flavonoids.

SEMIA587 ttsI mutant construction. Comparison of rhizobial tts sequences available in GenBank allowed primers to be designed corresponding to conserved regions of the gene (ttsI-FOR, 5' TCTGCCCGATGGAGACGGCTT-3', and ttsI-REV, 5' -TGCGCAGGCGATAGATCCCGA-3'). These primers were used to amplify an internal fragment of $t$ tsI using SEMIA587 genomic DNA as template. PCRs were performed in a $25 \mu \mathrm{l}$ volume, containing $50 \mathrm{ng}$ genomic DNA, 1 U Taq DNA polymerase (Life Technologies), $1 \times$ Taq DNA polymerase reaction buffer, $1.5 \mathrm{mM} \mathrm{MgCl}_{2}, 200 \mathrm{mM}$ dNTPs (Life Technologies) and $10 \mu \mathrm{mol}$ each primer. Amplification was carried out as follows: one initial denaturation step at $94{ }^{\circ} \mathrm{C}$ for $5 \mathrm{~min}, 30$ cycles of denaturation for $45 \mathrm{~s}$ at $94{ }^{\circ} \mathrm{C}$, annealing for $45 \mathrm{~s}$ at $52{ }^{\circ} \mathrm{C}$ and extension for $45 \mathrm{~s}$ at $72{ }^{\circ} \mathrm{C}$, and one final extension step for $5 \mathrm{~min}$ at $72^{\circ} \mathrm{C}$. Reactions were performed in a PCR Express temperature cycling system (Thermo Hybaid), and the fragments were visualized after electrophoresis at $80 \mathrm{~V}$ for $1 \mathrm{~h}$ on a $1.5 \%$ agarose gel stained with ethidium bromide. A molecular size marker (1 kb Plus DNA ladder, Gibco-BRL) was loaded onto all gels. The amplified fragment (364 bp) was cloned into the pGEM T-Easy vector (Promega), and sequenced in both the forward and the reverse 
direction with M13 universal primers in the ACTGene Laboratory [Centro de Biotecnologia, Universidade Federal do Rio Grande do Sul (UFRGS), RS, Brazil] using an ABI-PRISM 3100 automatic sequencer (Applied Biosystems). The sequence was checked and compared with those in GenBank using the BLAST program (http://blast.ncbi.nlm.nih. gov/Blast.cgi).

The $364 \mathrm{bp}$ amplified fragment containing the partial ttsI sequence was then subcloned into the EcoRI site of plasmid pK18mobsacB (Schäfer et al., 1994), generating pK18ttsI. This plasmid was linearized with $M l u \mathrm{I}$, and then overhanging ends were filled in with Klenow fragment (Invitrogen). The Omega cassette contained in pHP45 $\Omega$ (Prentki \& Kirsch, 1984) was removed with SmaI and inserted into the pK18ttsI MluI-linearized-filled-in plasmid, generating plasmid pK18ttsI $\Omega$. This plasmid was mobilized from E. coli DH5 $\alpha$ to strain SEMIA587 by triparental mating, using the helper plasmid pRK2013 (Figurski \& Helinski, 1979). Double recombination was selected by plating bacteria onto YEM plates containing the appropriate antibiotics (tetracycline and spectinomycin). Putative mutant strains (B. elkanii SEMIA587 ttsI) were confirmed by PCR and Southern blotting, using standard procedures (Sambrook \& Russell, 2001; data not shown).

Cloning of promoter sequences into the pPROBE NT reporter plasmid. The $t t s$ box motif identified in the promoter region of the SEMIA587 ttsI was isolated using a SiteFinding-PCR approach, as described by Tan et al. (2005). The $700 \mathrm{bp}$ fragment was cloned into the pGEM T-Easy vector and sequenced in both the forward and the reverse direction. The ttsI promoter region was PCR-amplified from pGEM T-Easy using pTtsI-sense (5'-GGGTCATCGCATTTTTCAAT$\left.3^{\prime}\right)$ and ttsI-REV primers. The PCR conditions were the same as described above. The $700 \mathrm{bp}$ amplified fragment was filled in with Klenow fragment and subcloned into the SmaI site of the broad-hostrange reporter vector pPROBE NT (Miller et al., 2000), generating plasmid pNTpttsI.

The $t$ s box motif from the nopC promoter region of B. elkanii SEMIA587 was amplified with primers pNopC-FOR (5'-TTGACGGTCGAGAGTTCTGT-3') and pNopC-REV (5'-CAGGACTTTTCCAGCTTTCA-3'), which were designed according to the B. elkanii USDA61 nopC sequence (GenBank accession no. FM162234). The amplified fragment $(\sim 1.0 \mathrm{~kb})$ was cloned into pGEM T-Easy and sequenced in both the forward and the reverse direction. The fragment was excised from pGEM T-Easy with HindIII and SalI and subcloned into pPROBE NT (also digested with HindIII and SalI), generating plasmid pNTpnopC.

The pPROBE NT constructs were mobilized into SEMIA587 and SEMIA587 ttsI by triparental matings using pRK2013 as the helper plasmid. Flavonoid induction assays were performed as described previously (Kobayashi et al., 2004; Wassem et al., 2008).

Measurements of GFP activity. $\mathrm{OD}_{595}$ and fluorescence (excitation filter at $485 \mathrm{~nm}$ and emission filter at $535 \mathrm{~nm}$ ) were measured on $100 \mu \mathrm{l}$ volumes of cultures $24 \mathrm{~h}$ post-induction using a Plate CHAMELEON multilabel detection platform (Hidex Oy). Optical densities and fluorescence were corrected to background levels using uninoculated media, and the results represent the means of at least three independent experiments. For the analysis, the fluorescence values obtained were normalized to the mean optical density at each time point. Data were statistically analysed using Student's $t$ test with a $P<0.05$ considered as significant.

Isolation and sequencing of SEMIA587 ttsI coding and promoter regions. To isolate a DNA fragment containing the complete ttsI ORF and upstream region from SEMIA587, primers were designed based on the sequence of the B. elkanii USDA61 tts cluster (GenBank accession no. FM162234). The primers NB-up
(5'-CGTTTTGAAGACCGTCAAGC-3'), NB-down (5'-ATTGCCGAAGTGGAATTGAC-3'), pTtsI-sense, TB-down (5'-GTCCATACACGCCAAGACCT- $3^{\prime}$ ) and TtsI-down (5'-TGTTGCTTTCGAGTTCGATTT-3') were used in several combinations (NB-up/NB-down, pTtsI-sense/TB-down, NB-up/TB-down, pTtsI-sense/TtsI-down). PCR conditions were as described above. All amplified fragments were cloned in pGEM-T Easy and sequenced in both the forward and the reverse direction with M13 universal primers.

Electrophoretic mobility shift assay (EMSA). The ttsI coding sequence (of SEMIA587) was PCR-amplified using primers TtsI-up (5'-GGTCATCGCATTTTTCAATC-3') and TtsI-down, using the proof-reading Platinum Pfx DNA Polymerase (Invitrogen) and the PCR conditions described above. The $900 \mathrm{bp}$ amplified DNA fragment was cloned into the vector pENTRTM/D-TOPO (Invitrogen), and sequenced to verify PCR fidelity. The insert was recombined into pGEX KG Gateway, using Gateway LR Clonase enzyme mix (Invitrogen), in $E$. coli $\mathrm{DH} 5 \alpha$ competent cells, generating pGEXTtsI. The recombinant plasmid was confirmed by PCR. pGEXTtsI was used to transform E. coli BL21 (Rosetta), and E. coli BL21 (Rosetta) carrying pGEXTtsI was induced with $0.5 \mathrm{mM}$ IPTG for $6 \mathrm{~h}$ at $28{ }^{\circ} \mathrm{C}$. Purification of the glutathione $S$-transferase (GST)-TtsI fusion was carried out using Glutathione Sepharose 4B from GE Healthcare Life Sciences. Protein concentration was assayed with Bradford reagent (BioAgency; Bradford, 1976).

A $55 \mathrm{bp}$ double-stranded oligonucleotide corresponding to the putative $t t s$ box from upstream of ttsI was obtained by annealing the sequences TBup (5'-AAGGCGGACGTCTGTCAGTTTCGTCGAACCTCTTGGTCAACCGTGACGCGCTTAA- $\left.3^{\prime}\right)$ and TBdo (5' TTAAGCGCGTCACGGTTGACCAAGAGGTTCGACGAAACTGACAGACGTCCGCCTT-3'). The same procedure, using sequences NBup (5'-ACGCCTATCCGCCTTTTGAATGCGTTCCATCAAAACAATTGATTTTGCCGATCCTGCA- ${ }^{\prime}$ ' and NBdo (5' ${ }^{\prime}$-TGCAGGATCGGCAAAATCAATTGTTTTGATGGAACGCATTCAAAAGGCGGATAGGCGT-3') was used to obtain a $58 \mathrm{bp}$ double-stranded oligonucleotide, which contained the nod box cis element. This fragment was used as a negative control. A second negative control used in this work was a $56 \mathrm{bp}$ double-stranded oligonucleotide containing the $t t s$ box upstream of the ttsI gene nucleotide modifications (TBmutUp, 5' -AAGGCGGACGTCTGGTTGTTTCGTCGAAACCTCTTGGTCAACCGTGACGCGCTTAA-3', and TBmutDo, 5' -TTAAGCGCGTCACGGTTGACCAAGAGGTTTCGACGAAACAA CCAGACGTCCGCCTT-3').

Binding conditions were assayed with the EMSA kit from Invitrogen as specified by the supplier. The double-stranded oligonucleotides were used at a concentration of $50 \mathrm{ng}$, and the purified TtsI-GST protein was tested at 50, 200 and $400 \mathrm{ng}$, for the tts box doublestranded oligonucleotide, and $200 \mathrm{ng}$ for the controls.

Analysis of secreted proteins. Extracellular proteins from SEMIA587 and its ttsI mutant strains, with or without flavonoid induction, were recovered from $50 \mathrm{ml}$ of RMS cultures grown in an orbital shaker (180 r.p.m.) for 72 h. Secreted proteins were extracted as described by Viprey et al. (1998). Aliquots of purified proteins were separated on $12 \%$ SDS-PAGE gels, using BenchMark Protein Ladder (Invitrogen) as a molecular mass standard, and stained with silver (Ausubel et al., 1994). For immuno detection, SDS-PAGE-separated proteins were transferred to PVDF Immobilon-P membranes (Millipore) and probed with antibodies raised against Rhizobium sp. NGR234 NopA and NopL proteins (1:2000 dilution). Horseradish peroxidase-labelled goat anti-rabbit immunoglobulin antibodies from the ECL kit (GE Life Sciences Amersham Pharmacia Biotech) were used as secondary antibodies.

Nodulation tests. Nodulation tests were performed in Magenta jars as described by Skorpil et al. (2005). All plants were grown at 
$18-28{ }^{\circ} \mathrm{C}$, with a photoperiod of $16 \mathrm{~h}$. Each plant was inoculated with $10^{7}$ bacteria and harvested 14 or 28 days after inoculation. Two plants were grown per Magenta jar; five jars were used for each condition tested. At harvest, total nodule number per jar was determined for each replicate. Only nitrogen-fixing (red) nodules were counted. Data from the nodulation tests (at a minimum in triplicate) were statistically analysed using Student's $t$ test with a $P<0.05$ considered significant. Plant seeds of G. $\max [(\mathrm{L}$.) Merrill] cv. McCall and cv. Peking, Macroptilium atropurpureum and Vigna unguiculata were obtained from LBMPS. Soybean G. $\max [($ L.) Merrill] cv. EMBRAPA 48 was obtained from Embrapa Soja. The seeds were surface-sterilized as previously described (Pueppke \& Broughton, 1999).

\section{RESULTS}

\section{Cloning of DNA sequences from the T3SS locus of SEMIA587}

A PCR-based approach was used to amplify the coding sequence of the $t t s I$ gene from SEMIA587 genomic DNA. The DNA sequences of all rhizobial ttsI genes were aligned to design specific primers from conserved regions for PCR. A product of the expected size ( $\sim 350 \mathrm{bp})$ was generated, cloned and verified to be highly homologous to ttsI by DNA sequencing (data not shown). The $t t s I$ fragment was used to create a ttsI mutant by disrupting the gene with an omega $(\Omega)$ cassette (see Methods for more details). The phenotype of the mutant was subsequently characterized (see below).

The ttsI promoter region was accessed by chromosome walking, using the SiteFinding-PCR approach (Tan et al., 2005). Analysis of the sequence obtained identified a potential $t t s$ box cis element $45 \mathrm{bp}$ upstream of the ttsI coding region (Fig. 1). No nod box was detected, but as the length of the sequence obtained was only $200 \mathrm{bp}$, a nod box motif could still be present further upstream. Nevertheless, the presence of the tts box motif in the promoter region of the B. elkanii SEMIA587 ttsI gene was unexpected, since this element has not been found in other ttsI promoter regions already sequenced.

\section{ctGTCAGttt gtcGaacctc ttggtcaacc $\quad 45 \mathrm{bp}$ tts \\ tcGTCAGctt gtcGaaActg atgcttccat $59 \mathrm{bp}$ nopC \\ tcgTCAGctT ntcGaaaGct annnctncta Consensus}

Fig. 1. Comparison of type III secretion (tts) box motifs found in the promoter regions of $B$. elkanii SEMIA587 tts/ and nopC genes with the tts box consensus sequence described for $B$. elkanii USDA61 T3SS genes (Okazaki et al., 2009). In the consensus sequence, all invariant nucleotides are shown in upper-case type. Lower-case type is used for nucleotides conserved in at least $50 \%$ of the sequences. Bold type in the tts box sequences of SEMIA587 indicates nucleotides that are identical to the consensus sequence. Numbers indicate distances in bp between the tts box and the assumed translational start site of the corresponding ORF.
Whilst this work was carried out, Okazaki et al. (2009) published the entire sequence of the T3SS cluster from another strain of B. elkanii, USDA61. Using the available data (GenBank accession no. FM162234), new primers were designed and used to amplify fragments of $t t s I$ and its regulatory regions from SEMIA587 genomic DNA. The amplified fragments covered a total of $2409 \mathrm{bp}$ of the ttsI region, including $1697 \mathrm{nt}$ upstream of the ttsI start codon. With this extra sequence of the $t t s I$ regulatory region, a nod box motif was found at a distance of $1621 \mathrm{bp}$ from the beginning of the $t t s I$ coding region. The presence of the $t t s$ box was confirmed $45 \mathrm{bp}$ from the ttsI coding region. The ttsI coding region was identified between nucleotides 1697 and 2386 of the sequenced region.

A second $t t s$ box was also isolated, from the promoter of a gene shown in other rhizobia to be under TtsI/tts box control (Wassem et al., 2008). The nopC tts box (59 bp upstream of the beginning of the nopC coding region) was obtained by PCR, using primers designed from the corresponding gene sequence of B. elkanii USDA61 (see Methods for more details). DNA sequence analysis confirmed the correct amplification of a $t$ ts box (Fig. 1).

\section{The flavonoids genistein and daidzein induce both the nopC and the ttsl promoter}

The promoter sequences of nopC and ttsI were subcloned into the pPROBE NT vector (Miller et al., 2000), and conjugated into the SEMIA587 wild-type and ttsI mutant, in order to test their transcription activation potential in the presence or absence of flavonoids. The flavonoids used were genistein and daidzein, which are known to be released by soybean. After $24 \mathrm{~h}$ of culture, SEMIA587 showed an elevated GFP fluorescence in the presence of the flavonoids only with the two plasmids containing the tts box promoter sequences upstream of GFP. The SEMIA587 ttsI mutant containing the same plasmids, however, did not display any significant fluorescence augmentation in $24 \mathrm{~h}$ grown cultures containing the same flavonoids (Fig. 2).

\section{EMSAs}

The presence of a $t t s$ box upstream of $t t s I$ in SEMIA587 was unusual. We decided to verify its functionality by demonstrating the binding of TtsI to this sequence with an EMSA. A TtsI-GST fusion protein was purified using Glutathione Sepharose 4B (GE Heathcare Life Sciences). The purification procedure was carried out according to the manufacturer's procedures. The TtsI-GST fusion was used in an EMSA, and Fig. 3 shows that the migration rate of the DNA fragment containing the tts box from the promoter region of $t t s I$ was delayed when it was incubated with two different amounts of the purified TtsI protein (200 and 400 ng; Fig. 3, lanes 5 and 6, respectively). Fifty nanograms of protein was not sufficient to delay the DNA fragment containing the $t t s$ box, however. The addition of $200 \mathrm{ng}$ of the same protein to control DNA sequences (i.e. 


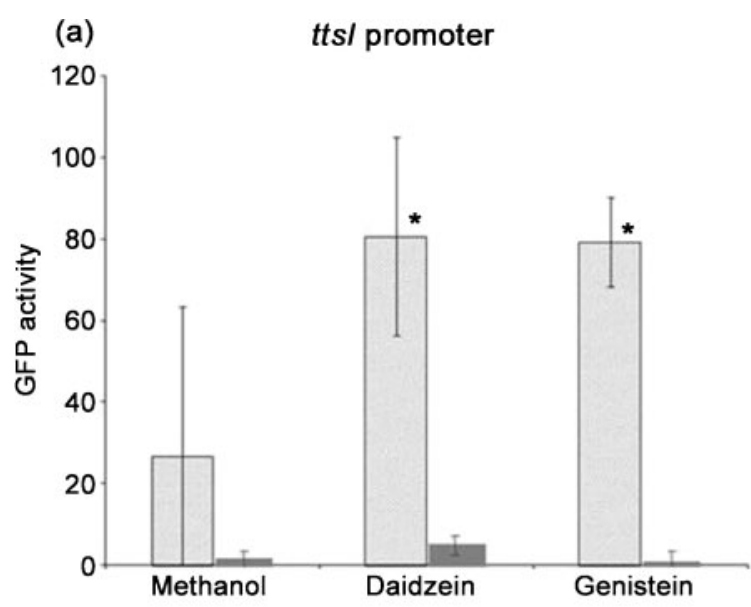

(b) nopC promoter

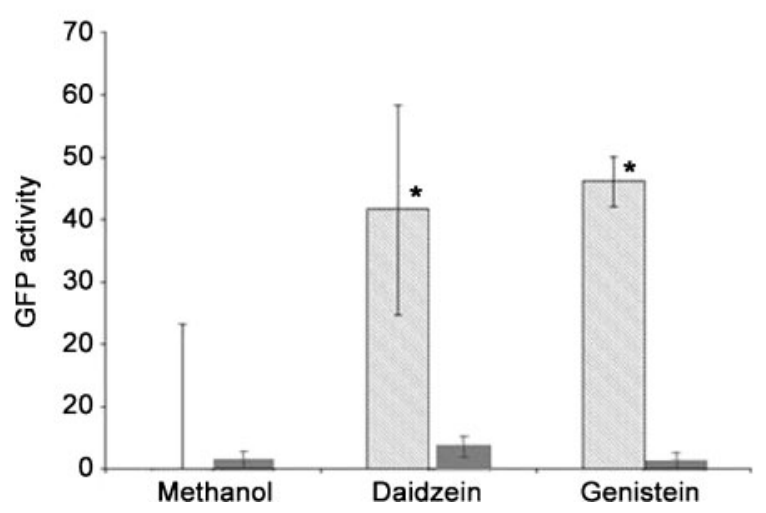

Fig. 2. Expression analysis of the $B$. elkanii SEMIA587 tts/ and nopC promoters. Fluorescence resulting from GFP activity was determined $24 \mathrm{~h}$ after induction with genistein or daidzein for strains carrying the promoter regions of the tts/ and nopC genes fused to the promoterless gfp gene. (a) tts box of the tts/ promoter region. (b) tts box of the nopC promoter region. Light-grey bars, constructions in the wild-type strain (B. elkanii SEMIA587); darkgrey bars, constructions in the mutant strain (B. elkanii SEMIA587 tts/). Asterisks indicate statistically significant differences (Student's $t$ test with $P<0.05$ ).

containing the nod box upstream of $t t s I$ or with a mutated version of the tts box; Fig. 3, lanes 8 and 10, respectively) caused no delay.

\section{Two Nops were not detected in culture supernatants of the $t t s /$ mutant}

The results of the GFP activity experiments showed that both daidzein and genistein could activate T3SS genes and that TtsI was required, since no induction was seen in the ttsI mutant. We thus tested whether these flavonoids could cause the appearance of extra proteins in culture supernatants of SEMIA587 and not in the SEMIA587 ttsI mutant. Cultures of both strains were induced with genistein and secreted proteins were isolated (see Methods). The

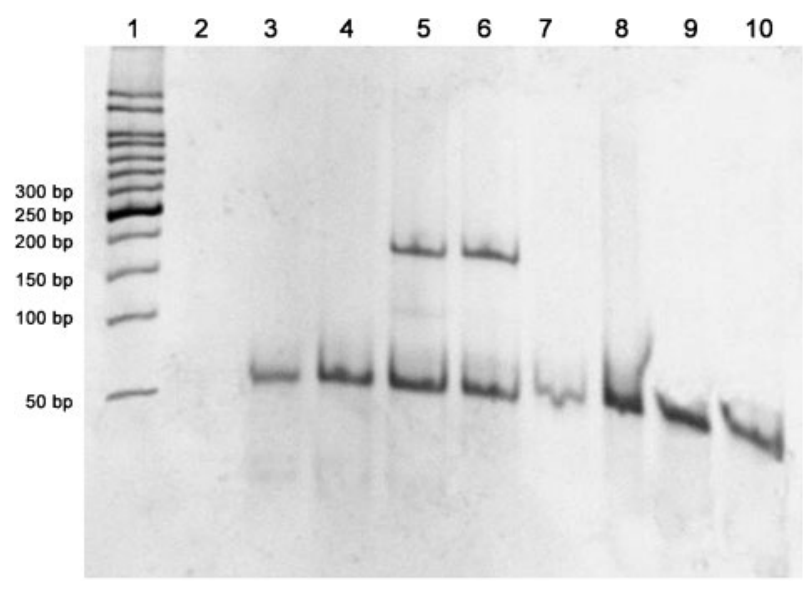

Fig. 3. Polyacrylamide gel (10\%) showing the binding of the Ttsl protein to the tts box promoter region of ttsl of $B$. elkanii SEMIA587. Lanes: 1, 50 bp Molecular Weight Marker (Ludwig Biotecnologia); 2, $200 \mathrm{ng}$ purified Ttsl; 3, $55 \mathrm{bp}$ fragment containing the tts box upstream of tts/; $4,55 \mathrm{bp}$ fragment containing the $t t s$ box upstream of $t t s /$ mixed with $50 \mathrm{ng}$ purified Ttsl protein; 5, 55 bp fragment containing the tts box upstream of tts/ mixed with $200 \mathrm{ng}$ purified Ttsl protein; 6, $55 \mathrm{bp}$ fragment containing the $t$ ts box upstream of $t \mathrm{ts} /$ mixed with $400 \mathrm{ng}$ purified Ttsl protein; 7, 58 bp fragment of the nod box upstream of $t t s / ; 8$, $58 \mathrm{bp}$ fragment of the nod box upstream of tts/ mixed with $200 \mathrm{ng}$ purified Ttsl protein; 9, $56 \mathrm{bp}$ fragment containing a mutated version of the tts box upstream of tts/; 10, $56 \mathrm{bp}$ fragment containing a mutated version of the tts box upstream of $t t s /$ mixed with $200 \mathrm{ng}$ purified Ttsl protein. The gel was stained with SYBR Green from the EMSA kit (Invitrogen) and visualized under UV.

supernatant protein extracts were separated by SDS-PAGE, but silver staining of these gels gave inconclusive results. Identical gels were then blotted onto membranes and tested with antibodies raised against known T3SS-secreted proteins, NopA and NopL, of Rhizobium sp. NGR234. The presence of these two Nops in culture supernatants of the wild-type strain was observed only in the presence of genistein. In the ttsI mutant, these proteins were not detected, even after flavonoid induction (Fig. 4).

\section{Nodulation tests show a B. elkanii T3SS host- dependent phenotype}

As previously demonstrated, the T3SSs of several rhizobial strains display a host-dependent phenotype (Viprey et al., 1998; Krause et al., 2002; Okazaki et al., 2009). Since the ttsI mutant of SEMIA587 was blocked for T3SS activity, comparison of this mutant with the wild-type in nodulation tests permitted the analysis of the role of the SEMIA587 T3SS in symbiosis. Three soybean cultivars, McCall, Peking and EMBRAPA 48, as well as Macroptilium atropurpureum (siratro) and Vigna unguiculata (cowpea), were tested. Soybean cultivars McCall and Peking were used, since it was shown that they have different responses to the S. fredii USDA257 T3SS (Meinhardt et al., 1993). 


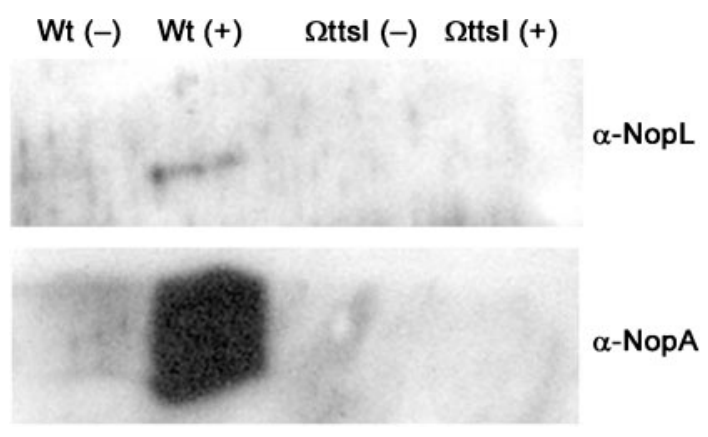

Fig. 4. Immunodetection of NopA and NopL proteins by the corresponding anti- $(\alpha-)$ NopA and $\alpha$-NopL (diluted $1: 2000$ ) antibodies in extracellular protein extracts of non-induced cultures $(-)$ and genistein-induced cultures (+). Wt, B. elkanii SEMIA587; $\Omega$ ttsl, B. elkanii SEMIA587 tts/ mutant strain. Samples were separated by $12 \%$ SDS-PAGE. The approximate sizes of the proteins are $25 \mathrm{kDa}$ for NopL and $7 \mathrm{kDa}$ for NopA.

EMBRAPA 48 is a modern commercial soybean cultivar extensively used by Brazilian soybean producers. Siratro and cowpea were used because of the observed effects of the B. japonicum USDA110 T3SS on the nodulation of these legumes (Krause et al., 2002).

Nodule numbers formed were assessed 14 and 28 days after plant inoculation with the wild-type and ttsI mutant strains (Fig. 5). For the soybean cultivars Peking and EMBRAPA 48, higher nodule numbers were observed when these plants were inoculated with the wild-type strain relative to the ttsI mutant. No significant differences in nodule number were observed with the soybean cultivar McCall or siratro. With cowpea, however, although no differences were observed 14 days post-inoculation, by 28 days the wild-type had induced significantly more nodules (Fig. 5).

\section{DISCUSSION}

T3SSs have been characterized from several rhizobia and shown to be important determinants of host range. These T3SSs are functional and capable of secreting numerous Nops (Deakin \& Broughton, 2009). The identification of T3SSs within B. elkanii has led to the investigation of their effects in the interaction of this key inoculant with soybean and other leguminous plants. Sequencing of the tts cluster of B. elkanii USDA61 has shown that it possesses many of the characteristics of other rhizobial T3SSs (Okazaki et al., 2009).

Generally, rhizobial T3SSs are controlled by NodD1 and activated when flavonoids are perceived (Kobayashi et al., 2004; Marie et al., 2004; Wassem et al., 2008). NodD1 induces the T3SS-specific regulator ttsI via a nod box in the promoter of ttsI (Viprey et al., 1998; Krause et al., 2002; Marie et al., 2004; López-Baena et al., 2008; Wassem et al., 2008). TtsI is able to activate other T3SS-related genes, as the promoters of tts genes share a common cis element, the tts box, to which TtsI binds (Krause et al., 2002). This element is conserved amongst all the T3SS-containing rhizobia (Marie et al., 2004; López-Baena et al., 2008; Wassem et al., 2008; Okazaki et al., 2009; Zehner et al., 2008). Analysis of the DNA sequence of the T3SS locus of B. elkanii USDA61 suggests a similar pattern of regulation, as $t$ tsI is preceded by a nod box, and other T3SS genes/ operons contain tts boxes in their promoters. No flavonoid activation was observed, however (Okazaki et al., 2009).

In this work we investigated the regulation of the T3SS of another B. elkanii strain, SEMIA587. Sequences corresponding to $t$ ts $I$ and $t$ ts box-containing promoters were identified and used to create a $t$ tsI mutant and reporter gene fusions, respectively. Two tts boxes, in the ttsI and nopC promoter regions, were both activated after $24 \mathrm{~h}$ of induction with genistein and daidzein, flavonoids known

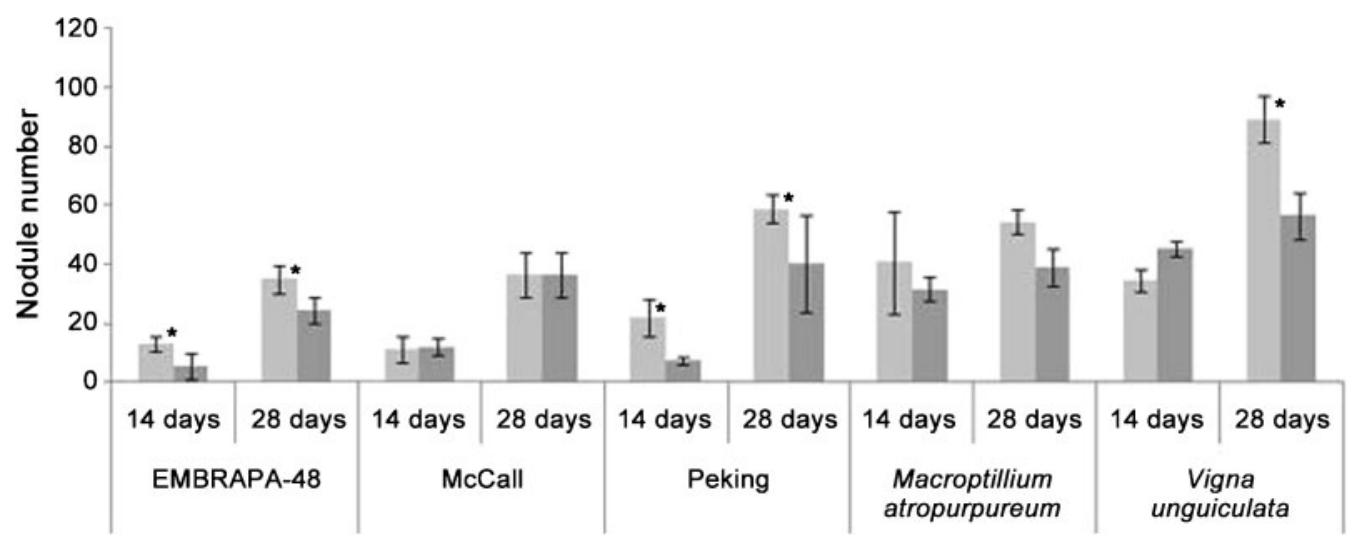

Fig. 5. Nodulation properties of $B$. elkanii SEMIA587 wild-type and tts/ mutant strains. Mature nodules were counted after 14 and 28 days. Mean values were determined from three plants in at least three independent tests; error bars, SD. Light-grey bars, B. elkanii SEMIA587 wild-type strain; dark-grey bars, B. elkanii SEMIA587 tts/ mutant strain. EMBRAPA 48, McCall and Peking are soybean $\{$ G. $\max [($ L.) Merrill]\} cultivars. Asterisks denote statistically significant differences (Student's $t$ test with $P<0.05$ ). 
to be released by soybean. Furthermore, this activation required a functional $t t s I$ gene, since no induction was seen with a ttsI mutant. Thus, the T3SS of SEMIA587 can be induced by soybean-derived flavonoids, and appears to follow the standard rhizobial mechanism, i.e. TtsI controlling $t t s$ box-containing promoters.

The promoter region of ttsI from SEMIA587 is unusual, however, as it contains a $t$ s box. A fragment of the promoter sequence carrying this $t t s$ box (fused to GFP) was shown to be flavonoid-inducible (see above), and thus we characterized this tts box further. An EMSA was performed, and showed that TtsI was able to bind to this tts box. One interpretation of the presence of a functional $t t s$ box upstream of $t t s I$ would be to reinforce the activation of $t$ tsI transcription after flavonoid induction. Such a potential positive-feedback loop implies that in SEMIA587, the regulation of T3SS genes is markedly different from that of other T3SS-possessing rhizobia. The coding and regulatory regions of ttsI from SEMIA587 were almost identical to those from B. elkanii USDA61 described by Okazaki et al. (2009). Those authors identified nine $t t s$ box elements in the sequenced region of B. elkanii USDA61, including one upstream of ttsI but even more distal than the nod box identified 1627 bp from ttsI. The $t t s$ box identified and characterized in the present work is located only 45 bp from ttsI (see Fig. 1).

We performed a protein secretion assay with cultures of SEMIA587 wild-type and ttsI mutant strains in the presence of genistein. Two Nops (NopA and NopL) were not detectable in culture supernatants of the ttsI mutant strain, whilst the wild-type strain was able to secrete both proteins, although only in the presence of the flavonoid. Similar results were obtained when an S. fredii HH103 ttsI mutant strain was tested for Nop secretion (López-Baena et al., 2008). Therefore, inactivation of the ttsI gene blocks Nop secretion and/or production in these bacteria.

Okazaki et al. (2009) did not find any difference in protein secretion when they analysed the USDA61 strain of $B$. elkanii in the presence or absence of the same flavonoid used in our work (genistein). Nops in culture supernatants could only be identified from B. elkanii USDA61 when comparisons were made between the wild-type strain and a mutant in a T3SS gene $(r h c J)$ which encodes an essential structural component of the machinery. The discrepancy between the results obtained by Okazaki et al. (2009) and those reported in this work could be due to the culture medium used in the two experiments. Whilst in the present work RMS medium was used, Okazaki et al. (2009) used arabinose gluconate medium (Sadowsky et al., 1987). It has been shown that Nop secretion can be influenced by some compounds of the culture medium. For example, Krishnan et al. (2007) have shown that calcium prevents the accumulation of NopB and NopA, and drastically reduces that of NopX and NopL in S. fredii USDA257. It would be interesting to determine whether $B$. elkanii USDA61 secretes NopA and NopL when cultured in RMS medium, and whether the addition of flavonoids has any effect.
Nodulation tests were performed with SEMIA587 and a derivative $t t s$ mutant strain on three soybean cultivars (EMBRAPA 48, McCall and Peking), as well as $M$. atropurpureum and $V$. unguiculata. Characterization of the resulting plants showed that the number of nodules formed by soybean cultivars EMBRAPA 48 and Peking was affected when $t t s I$ was inactive, particularly at 14 days postinoculation. Nodule formation by cultivar Peking is thus enhanced by the presence of an active T3SS in SEMIA587 as well as in S. fredii strains USDA257 and HH103. The number of nodules formed by cowpea was significantly higher when plants were inoculated with the wild-type strain only after 28 days; there was no difference after 14 days. Nodulation tests performed with a $B$. japonicum USDA110 ttsI mutant and soybean have shown that there is a statistically significant difference in the number of nodules 10 days after inoculation, although this phenotype is no longer significant after 20 days of inoculation (Krause et al., 2002). Although there were no obvious effects of the SEMIA587 T3SS on McCall (unlike S. fredii USDA257) and siratro (as for other T3SS-utilizing rhizobia), on some legumes an active T3SS of SEMIA587 appeared to increase the number (and the speed of formation) of nodules. A T3SS host-dependent phenotype has also been observed with B. elkanii USDA61 and derivative $r h c C 2$ and $r h c J$ mutants (Okazaki et al., 2009), where nodule formation differs according to the cultivar of soybean (Hill or Clark in that case) or Vigna radiata (CN36 or KPS1). With M. atropurpureum, the T3SS mutant strains induced fewer but larger nodules than the wild-type strain (Okazaki et al., 2009).

The B. elkanii USDA61 rhcC2 and rhcJ mutants blocked Nop secretion alone. In the case of SEMIA587, although the ttsI mutant could not secrete Nops, an alternative possibility for the plant phenotypes that we cannot rule out is that TtsI in SEMIA587 might regulate other symbiotically relevant genes besides the T3SS. As well as the T3SS, TtsI of Rhizobium sp. NGR234 also activates the production of a new lipopolysaccharide species which is a key signal for nodule formation by some legumes (Marie et al., 2004). As no further information for the genome of SEMIA 587 is available, this hypothesis remains to be verified.

In conclusion, our work demonstrates the importance of the T3SS of SEMIA587 in the establishment of symbiosis with legumes. As for B. elkanii USDA61, the transcriptional mechanisms for the activation of the T3SS differ from the rhizobial paradigm. In USDA61 no flavonoid induction is observed, whereas for SEMIA587 the potential for a flavonoid-induced positive feedback activation of the T3SS exists. SEMIA587 is an important inoculant for the Brazilian soybean crop and we demonstrate that functions of the SEMIA587 TtsI have a positive role in the formation of nodules on one crop cultivar of soybean, probably by activating the T3SS. Future work will determine whether nodulation of other Brazilian soybean varieties is similarly improved, which rhizobial effectors are responsible for the improvement, and whether there is continual production of these effectors during the SEMIA587-soybean interaction. 


\section{ACKNOWLEDGEMENTS}

The authors are grateful to Fundação Estadual de Pesquisa Agropecuária (FEPAGRO) for providing the B. elkanii SEMIA587 strain, to Dr Carmem Gottfried from Laboratório de Bioquímica, UFRGS, for help and support in the GFP assay, to Dr Henrique B. Ferreira from Departamento de Biologia Molecular e Biotecnologia, UFRGS, for help in the TtsI-GST purification, and to Empresa Brasileira de Pesquisa Agropecuária (EMBRAPA) - Soja for the seeds of the EMBRAPA 48 soybean cultivar. This work was supported by a grant and fellowships (in Brazil and Switzerland for S. B.C.) from Conselho Nacional de Desenvolvimento Científico e Tecnológico $(\mathrm{CNPq} /$ Brazil). We are indebted to Y.-Y. Aung and D. Gerber (Geneva University) for their help with this work. Financial support from the Fonds National Suisse de la Recherche Scientifique (projects 3100AO-104097 and 3100A0-116858) to W.J.D. and W.J.B. is gratefully acknowledged.

\section{REFERENCES}

Ausubel, F. M., Brent, R., Kingston, R. E., Moore, D. D., Seidman, J. G., Smith, J. A. \& Struhl, K. (editors) (1994). Current Protocols in Molecular Biology. New York: Wiley.

Bartsev, A. V., Boukli, N. M., Deakin, W. J., Staehelin, C. \& Broughton, W. J. (2003). Purification and phosphorylation of the effector protein NopL from Rhizobium sp. NGR234. FEBS Lett 554, 271-274.

Bartsev, A. V., Deakin, W. J., Boukli, N. M., McAlvin, C. B., Stacey, G., Malnoe, P., Broughton, W. J. \& Staehelin, C. (2004). NopL, an effector protein of Rhizobium sp. NGR234, thwarts activation of plant defence reactions. Plant Physiol 134, 871-879.

Bradford, M. M. (1976). A rapid and sensitive method for the quantification of microgram quantities of protein utilizing the principle of protein-dye binding. Anal Biochem 72, 248-254.

Broughton, W. J., Wong, C. H., Lewin, A., Samrey, U., Myint, H., Meyer, Z. A. H., Dowling, D. N. \& Simon, R. (1986). Identification of Rhizobium plasmid sequences involved in recognition of Psophocarpus, Vigna, and other legumes. J Cell Biol 102, 1173-1182.

Deakin, W. J. \& Broughton, W. J. (2009). Symbiotic use of pathogenic strategies: rhizobial protein secretion systems. Nat Rev Microbiol 7, 312-320.

Ferreira, M. C., Andrade, D. S., Chueire, L. M. O., Takemura, S. M. \& Hungria, M. (2000). Tillage method and crop rotation effects on the population sizes and diversity of bradyrhizobia nodulating soybean. Soil Biol Biochem 32, 627-637.

Figurski, D. H. \& Helinski, D. R. (1979). Replication of an origincontaining derivative of plasmid RK2 dependent on a plasmid function provided in trans. Proc Natl Acad Sci U S A 76, 1648-1652.

Freiberg, C., Fellay, R., Bairoch, A., Broughton, W. J., Rosenthal, A. \& Perret, X. (1997). Molecular basis of symbiosis between Rhizobium and legumes. Nature 387, 394-401.

Hueck, C. J. (1998). Type III protein secretion systems in bacterial pathogens of animals and plants. Microbiol Mol Biol Rev 62, 379-433.

Kobayashi, H., Graven, Y. N., Broughton, W. J. \& Perret, X. (2004). Flavonoids induce temporal shifts in gene-expression of nod-box controlled loci in Rhizobium sp. NGR234. Mol Microbiol 51, 335-347.

Krause, A., Doerfel, A. \& Göttfert, M. (2002). Mutational and transcriptional analysis of the type III secretion system of Bradyrhizobium japonicum. Mol Plant Microbe Interact 15, 1228-1235.

Krishnan, H. B., Kim, W. S. \& Sun-Hyung, J. (2007). Calcium regulates the production of nodulation outer proteins (Nops) and precludes pili formation by Sinorhizobium fredii USDA257, a soybean symbiont. FEMS Microbiol Lett 271, 59-64.
Lan, C.-Y. \& Igo, M. M. (1998). Differential expression of the OmpF and OmpC porin proteins in Escherichia coli K-12 depends upon the level of active OmpR. J Bacteriol 180, 171-174.

López-Baena, F. J., Vinardell, J. M., Pérez-Montano, F., CrespoRivas, J. C., Bellogín, R. A., Espuny, M. R. \& Ollero, F. J. (2008). Regulation and symbiotic significance of nodulation outer proteins secretion in Sinorhizobium fredii HH103. Microbiology 154, 18251836.

Marie, C., Broughton, W. J. \& Deakin, W. J. (2001). Rhizobium type III secretion systems: legume charmers or alarmers? Curr Opin Plant Biol 4, 336-342.

Marie, C., Deakin, W. J., Viprey, V., Kopcinska, J., Golinowski, W., Krishnan, H. B., Perret, X. \& Broughton, W. J. (2003). Characterization of Nops, nodulation outer proteins, secreted via the type III secretion system of NGR234. Mol Plant Microbe Interact 16, 743-751.

Marie, C., Deakin, W. J., Ojanen-Reuhs, T., Diallo, E., Reuhs, B., Broughton, W. J. \& Perret, X. (2004). TtsI, a key regulator of Rhizobium species NGR234 is required for type III-dependent protein secretion and synthesis of rhamnose-rich polysaccharides. Mol Plant Microbe Interact 17, 958-966.

Meinhardt, L. W., Krishnan, H. B., Ballati, P. A. \& Pueppke, S. G. (1993). Molecular cloning and characterization of a sym plasmid locus that regulates cultivar-specific nodulation of soybean by Rhizobium fredii USDA257. Mol Microbiol 9, 17-29.

Miller, W. G., Leveau, J. H. J. \& Lindow, S. E. (2000). Improved $g f p$ and inaZ broad-host-range promoter-probe vectors. Mol Plant Microbe Interact 13, 1243-1250.

Okazaki, S., Zehner, S., Hempel, J., Lang, K. \& Gottfert, M. (2009). Genetic organization and functional analysis of the type III secretion system of Bradyrhizobium elkanii. FEMS Microbiol Lett 295, 88-95.

Pallen, M. J., Chaudhuri, R. R. \& Henderson, I. R. (2003). Genomic analysis of secretion systems. Curr Opin Microbiol 6, 519-527.

Pueppke, S. G. \& Broughton, W. J. (1999). Rhizobium sp. strain NGR234 and $R$. fredii USDA257 share exceptionally broad, nested host ranges. Mol Plant Microbe Interact 12, 293-318.

Prentki, P. \& Kirsch, H. M. (1984). In vitro insertional mutagenesis with a selectable DNA fragment. Gene 29, 303-313.

Rumjanek, N. G., Dobert, R. C., van Berkum, P. \& Triplett, E. W. (1993). Common soybean inoculant strains in Brazil are members of Bradyrhizobium elkanii. Appl Environ Microbiol 59, 4371-4373.

Sadowsky, M. J., Tully, R. E., Cregan, P. B. \& Keyser, H. H. (1987). Genetic diversity in Bradyrhizobium japonicum serogroup 123 and its relation to genotype-specific nodulation of soybean. Appl Environ Microbiol 53, 2624-2630.

Sambrook, J. \& Russell, D. W. (2001). Molecular Cloning: a Laboratory Manual. Cold Spring Harbor, NY: Cold Spring Harbor Laboratory.

Schäfer, A., Tauch, A., Jäger, W., Kalinowski, J., Thierbach, G. \& Pühler, A. (1994). Small mobilizable multipurpose cloning vectors derived from the Escherichia coli plasmids pK18 and pK19: selection of defined deletions in the chromosome of Corynebacterium glutamicum. Gene 145, 69-73.

Skorpil, P., Saad, M. M., Boukli, N. M., Kobayashi, H., Ares-Orpel, F., Broughton, W. J. \& Deakin, W. J. (2005). NopP, a phosphorylated effector of Rhizobium sp. strain NGR234, is a major determinant of nodulation of the tropical legumes Flemingia congesta and Tephrosia vogelii. Mol Microbiol 57, 1304-1317.

Tan, G., Gao, Y., Shi, M., Zhang, X., He, S., Chen, Z. \& An, C. (2005). SiteFinding-PCR: a simple and efficient PCR method for chromosome walking. Nucleic Acids Res 33, e122. 
Vincent, J. M. (1970). A Manual for the Practical Study of Root Nodule Bacteria. Oxford: Blackwell Scientific.

Viprey, V., Del Greco, A., Golinowski, W., Broughton, W. J. \& Perret, X. (1998). Symbiotic implications of type III protein secretion machinery in Rhizobium. Mol Microbiol 28, 13811389.

Wassem, R., Kobayashi, H., Kambara, K., Le Quéré, A., Walker, G. C., Broughton, W. J. \& Deakin, W. J. (2008). TtsI regulates symbiotic genes in Rhizobium species NGR234 by binding to tts boxes. Mol Microbiol 68, 736-748.

Zehner, S., Schober, G., Wenzel, M., Lang, K. \& Gottfert, M. (2008). Expression of the Bradyrhizobium japonicum type III secretion system in legume nodules and analysis of the associated $t$ ts box promoter. Mol Plant Microbe Interact 21, 1087-1093.

Edited by: I. K. Toth 